\title{
A Prediction Model for Electromagnetic Radiation of Multi- system Base Station
}

\author{
Yang Jie, Wang Lei, Li Xianli, Wu Jie \\ Department of Communication Command \\ Chongqing Communication College, \\ Chongqing, 400035, China \\ e-mail:yangjie5581@163.com
}

\author{
Chao Yongjin, Pan Cunzhen \\ Station of Environment Radiation Supervision \\ Chongqing Environmental Protection Agency \\ Chongqing, 400015, China \\ e-mail:773783896@qq.com
}

\begin{abstract}
In order to understand the distribution feature of the electromagnetic radiation in nearby regions around the Multi-system mobile communication base stations, a theoretical prediction model of electromagnetic radiation at multi-system base station is proposed, based on the distribution characteristic of electromagnetic power for the base station antenna. Though antenna characteristic parameters, such as the normalized directivity function, antenna gain, gain of the array element, shaped gain and so on, the distribution of its power density of the Multi-radiation source in Multi- system base station is calculated. Compare with the surveys and analysis of the actual electromagnetic radiation and environment, the result has showed this electromagnetic radiation at multi-system base station was scientific and rational.
\end{abstract}

Keywords-Multi-system Base Station; Electromagnetic Radiation; Antenna Characteristic; Prediction Model

\section{INTRODUCTION}

To conserve land, reduce energy and raw material consumption, the mobile communication base station is building and sharing to improve the utilization of telecommunications infrastructure [1]. At the same time, the antenna generates electromagnetic radiation in the sharing site is relatively concentrated, which will increase in a single physical site ambient electromagnetic radiation levels. And the base station's building and sharing is related to $2 \mathrm{G}$ systems (GSM, DCS, CDMA1X) and 3G systems (TDSCDMA, CDMA2000, WCDMA) [2]. Because of the complex of electromagnetic radiation in the sharing base station, the research of space model of the electromagnetic radiation in the sharing base station is greater difficulties. So research of base station building and sharing focused on the development of strategy, policy research, implementation, problems and regulatory strategies and so on. But the analysis of electromagnetic radiation distribution, superimposed impact, the smart antenna in sharing base station is not yet mature, it is necessary to carry out a useful discussion in this regard [3-6].

Though sharing, close-set or multiple sets of launch systems, the probability of excessive electromagnetic radiation is greatly increased. So it is important to research on the prediction and assessment of electromagnetic radiation in the shared base station and multi-radiation source scene, and to ensure the electromagnetic radiation of sharing mobile communication base station meet the current requirements of environmental standards. In this paper, the prediction model of the ambient electromagnetic radiation on the multi-network is proposed, a scientific assessment of the shared mobile communication base station on the surrounding environment is given, and the analysis and comparison of the predicted and measured data is analyzed and compared. Then the method to reduce electromagnetic radiation in the sharing mobile communication base stations is presented.

\section{THE STANDARD FOR FAR-FIELD REGION}

Accordance with the Maxwell electromagnetic field theory, whether the public activity area can be far-field region is calculated by following formula to determine:

$$
R \geq \frac{2 D^{2}}{\lambda}
$$

Wherein $\mathrm{R}$ is the distance from an electromagnetic radiation source to the zone of the public activity, in units of $\mathrm{m}$; $\mathrm{D}$ is maximum length of the antenna or a radiator, the unit is $m$; $\lambda$ for the wavelength of the electromagnetic radiation; such as digital communication network GSM, its uplink frequency band is $900 \mathrm{MHz}$, downlink frequency is 1800 $\mathrm{MHz}$, so emission wavelength is $0.3 \mathrm{~m}$ approximately, the maximum length of the antenna is $1.30 \mathrm{~m}$, and the far-field region is about $10 \mathrm{~m}$ away from the antenna distance available.

\section{THE THEORETICAL PREDICTION MODEL}

In a mobile communication system, the base station antenna is a major source of electromagnetic radiation. By which, the electromagnetic signal is transmitted and received. Any antennas have some certain characteristic parameters, and the characteristics of the antenna parameters, including transmit power, antenna gain, the half power angle, electronic inclination and so on. Though these characteristic parameters, the performance of the antenna can be evaluated and calculated. Then combine with the antenna engineering theory, the electromagnetic radiation formula to a base station antenna can be received. So a simulation model of electromagnetic radiation in base station is presented.

Because of the lots of reflecting objects around the base station, the impact from the ground reflected wave in the vicinity of the inspection points as well, the general freespace propagation model will have some differences with the actual measurements. But away from the ground, not too far from the antenna (in the case of the far-field area), and there 
is no obvious reflector around surrounding, then the free space propagation model may be used to calculation.

\section{A. The Prediction model of single radiation source}

If the feed-in power of transmitting antennas is $\mathrm{P}(\mu \mathrm{W})$ in the antenna port and gain is $G$ for the specific direction, the distance from receiver to antenna phase center is $\mathrm{r}(\mathrm{m})$. Then the power flux density of receiving point:

$$
\mathrm{S}\left(\frac{\mu \mathrm{W}}{\mathrm{cm}^{2}}\right)=\frac{\mathrm{PG}}{4 \pi \mathrm{r}^{2}} \times 100
$$

Where, $\mathrm{P}$ is mean power of the transmitter (Unit : $\mu \mathrm{W}$ ), $\mathrm{G}$ is antenna gain (multiple), $\mathrm{r}$ is distance $(\mathrm{m})$ between the antenna and the measured point.

Combined with the dependent variable parameters, such as actual antenna transmitting power, gain, half power angle of electrical tilt, etc, antenna engineering theory is applied to the formula transformation. And a base station electromagnetic radiation simulation model is established though directivity function has been normalized and deduced Taking into account the far-field region direct wave only, the power density calculation method of antenna electromagnetic radiation is showed as formula (2). To calculate the electromagnetic radiation in arbitrary point of the antenna, the antenna directivity function must be considered, thus the single radiation source antenna electromagnetic radiation power density prediction models can be derived, that is:

$$
\mathrm{S}\left(\frac{\mu \mathrm{W}}{\mathrm{cm}^{2}}\right)=\frac{\mathrm{P} \cdot \mathrm{G} \cdot \mathrm{F}(\varphi)^{2} \cdot \mathrm{F}(\theta)^{2}}{4 \pi \mathrm{r}^{2}} \times 100
$$

Where, $S$-power density of the electromagnetic radiation $\left(\mu \mathrm{W} / \mathrm{cm}^{2}\right)$;

$\mathrm{P}$-antenna transmitting power ( W ) ;

G-antenna gain $(\mathrm{dB})$;

$F(\varphi) 、 F(\theta)$ is value in the prediction of the direction of the antenna in the horizontal plane and vertical plane normalized directional function respectively;

-distance from the forecast point to the transmitting antenna center point $(\mathrm{m})$.

As in [7], the antenna with 65 degree angle in GSM network:

$$
\mathrm{F}(\varphi)=-0.00235 \times \varphi^{2}
$$

In the formula (4), relative to the antenna main lobe axis (horizontal), $\varphi$ is the azimuth angle of the forecast point. And the vertical directivity function of the antenna is showed as a directivity function of uniform linear array accordingly. Then the analytic formula is:

$$
F(\theta)=\frac{\sin \left(\sin \theta \cdot \frac{160^{\circ}}{2 \theta}\right)}{\frac{\sin \theta 160^{\circ}}{2 \theta}}
$$

For the TD-SCDMA system base station, due to the adoption of smart antenna, the calculation of the electromagnetic radiation level varies greatly, compared with other mobile communication system. In smart antenna system, each user is tracked by beam. When the user moves, the direction of the beam is changed. Therefore there is no fixed main radiation direction, i.e. the direction of maximum radiation of electromagnetic radiation. While the system is under full load, and all users are concentrated in the same position, the electromagnetic radiation of the direction is maximum value. At this time, all of the array element radiation energy is concentrated in this position, shaped gain maximum. The frequency of TD-SCDMA system is 2010 $\mathrm{MHz} 2025 \mathrm{MHz}$, using time division duplex technology, i.e. the uplink and downlink work on the same frequency. Considering the difference between uplink and downlink, calculation formula modified by formula (2) is [8]:

$$
\mathrm{S}\left(\frac{\mu \mathrm{W}}{\mathrm{cm}^{2}}\right)=\frac{\mathrm{P}_{\mathrm{k}} \mathrm{G}_{\mathrm{k}} \mathrm{G}_{\mathrm{w}}}{4 \pi \mathrm{r}^{2}} \mathrm{D}_{\mathrm{u}} \times 100
$$

In which, $P_{k}$ is array transmitting power, $G_{k} 、 G_{w}$ are array gain and shaped gain respectively, $D_{u}$ is the ratio of downlink time slot in the sub frame(duty ratio of time slot ).

\section{B. Prediction model of multiple radiations}

When the mobile communication system base stations share a site, the site may has a good number of antennas (radiation sources). Because of the need to estimate the maximum value of electromagnetic radiation, consider of these antennas (radiation sources) placed together. In practical engineering application, these antennas in the sharing site can't be placed together completely, but in the vertical direction or the horizontal direction, each other will have a certain spatial isolation.

If the base station has a total of $\mathrm{n}$ transmit antenna to sharing, the maximum output power of the transmit antenna is $\mathrm{Pi}(\mathrm{W})$, and the gain is $\mathrm{Gi}(\mathrm{dB})$. And the antenna radiation is not in the same direction, the power flux density in the point is showed as formula (7), where the distance between the point and the phase center of transmitting antenna is $r(\mathrm{~m})$ :

$$
S\left(\frac{W}{m^{2}}\right)=\sum_{i=1}^{n} \frac{P_{i} \cdot G_{i} \cdot F_{i}(\varphi)^{2} \cdot F_{i}(\theta)^{2}}{4 \pi r_{i}^{2}}
$$

Where, $\mathbf{S}$ is total power density of the multiple radiations electromagnetic radiation $\left(\mathrm{W} / \mathrm{cm}^{2}\right)$;

$\mathrm{P}_{\mathrm{i}}$ - power of the antenna $\mathrm{i}(\mathrm{W})$ in transmitting

$\mathrm{G}_{\mathrm{i}}$-gain $(\mathrm{dB})$ for antennas $\mathrm{i}$

$F_{i}(\varphi) 、 F_{i}(\theta)$ is the value in the prediction of the direction of the antenna in the horizontal plane and vertical plane normalized directional function respectively.

$r_{i}$-the distance between the forecast point to the transmitting antenna i center point (m).

In fact, the unit of total power density is too large. For ease of use in engineering, it can also be expressed as the formula:

$$
S\left(\frac{\mu W}{\mathrm{~cm}^{2}}\right)=\sum_{i=1}^{n} \frac{100 P_{i} \cdot G_{i} \cdot F_{i}(\varphi)^{2} \cdot F_{i}(\theta)^{2}}{4 \pi r_{i}^{2}}
$$

To a certain extent, this way that the base stations share one site can reduce difficulty of the base station location, save the construction cost and maintenance cost, and so on. But according to the calculation result of the prediction model, the base station sharing site will result in the increase of partial region of electromagnetic radiation intensity. Those may be influence the zone which is thought to be safe originally, and may affect greatly electromagnetic 
environment in the area. Therefore, additional antennas (radiation sources) must be assessed adequately on the surrounding electromagnetic environment effects, and the number antenna sharing site is increased by reasonable measures and ways.

\section{DATA ANALYSIS}

For example, a mobile typical base station of a communication company, calculated the influence on the surrounding environment due to mobile GSM communication network and network for $3 \mathrm{G}$ in common base station, and calculated the influence of electromagnetic radiation on the surrounding environment due to both the single station, and Multi-network sharing mobile communication base station, in the effect prediction model.

For a single WCDMA base station, its operating frequency is $2140 \mathrm{MHz}$, antenna length $1.3 \mathrm{~m}$, normal power $10 \mathrm{~W}$, maximum output power $20 \mathrm{~W}$, and antenna gain $17.5 \mathrm{dBi}$. So test the electromagnetic radiation of single base station in the normal power and maximum power state. Then calculate the power density values of the WCDMA base station in single base station prediction model, as shown in Table 1, and the base station measured results, as shown in Table 2.

TABLE I. Power Density Prediction Values

\begin{tabular}{|c|c|c|}
\hline \multirow{2}{*}{ Distance/m } & \multicolumn{2}{|c|}{ Power Density Prediction Values/ $\left(\boldsymbol{\mu W} / \mathbf{c m}^{2}\right)$} \\
\cline { 2 - 3 } & Normal Power(10W) & Maximum Power(20W) \\
\hline 5 & 75.21 & 148.64 \\
\hline 10 & 18.80 & 37.16 \\
\hline 15 & 8.36 & 16.52 \\
\hline 20 & 4.70 & 9.29 \\
\hline 25 & 3.01 & 5.94 \\
\hline 30 & 2.09 & 4.13 \\
\hline 35 & 1.53 & 3.03 \\
\hline 40 & 1.17 & 2.32 \\
\hline 45 & 0.93 & 1.83 \\
\hline 50 & 0.75 & 1.48 \\
\hline
\end{tabular}

TABLE II. WCDMA Base Station Measured Results

\begin{tabular}{|c|c|c|c|}
\hline \multirow{2}{*}{ serial number } & \multirow{2}{*}{ Distance/m } & \multicolumn{2}{|c|}{ Measured Results $/\left(\boldsymbol{\mu W} / \mathbf{c m}^{2}\right)$} \\
\cline { 3 - 4 } & & $\begin{array}{c}\text { Transmit } \\
\text { Power(10W })\end{array}$ & $\begin{array}{c}\text { Transmit } \\
\text { Power(20W })\end{array}$ \\
\hline 1 & 5 & 12.37 & 148.64 \\
\hline 2 & 10 & 27.43 & 37.16 \\
\hline 3 & 15 & 6.76 & 16.52 \\
\hline 4 & 20 & 4.09 & 9.29 \\
\hline 5 & 25 & 1.23 & 5.94 \\
\hline 6 & 30 & 3.28 & 4.13 \\
\hline 7 & 35 & 0.75 & 3.03 \\
\hline 8 & 40 & 1.34 & 2.32 \\
\hline 9 & 45 & 2.09 & 1.83 \\
\hline 10 & 50 & 0.55 & 1.48 \\
\hline
\end{tabular}

Multi-systems base-station, including $2 \mathrm{G}$ and $3 \mathrm{G}$ systems shared base station. GSM band: Uplink: 890 909MHz, Downlink: 935 954MHz. 3G band: Uplink: 1920 1980MHz Downlink: $2110 \sim 2170 \mathrm{MHz}$.

In the worst of situations, that the directions of maximum radiation were the same, GSM antenna and $3 \mathrm{G}$ antenna main ray direction completely overlapped. In this case, it could be regarded as the same radiator transmits different frequency electromagnetic waves. That is, when the base station sharing sites, one site would have multiple sources of radiation. Taking into account the maximum impact of the pollution sources of electromagnetic radiation environment around, assuming a plurality of radiation source placed at the same position, and the maximum radiation direction was the same direction, when evaluated. In this example, the power density of electromagnetic radiation was the sum of both the GSM system and the $3 \mathrm{G}$ system. Then get prediction results of the electromagnetic radiation, using multi radiation source prediction model, as shown in Table 3. And get the measured results of the electromagnetic radiation, using multi radiation source monitoring means, as shown in Table 4.

TABLE III. Electromagnetic Radiation Prediction Results

\begin{tabular}{|c|c|c|}
\hline serial number & $\begin{array}{c}\text { Axial Distance } \\
\text { from Antenna/m }\end{array}$ & Power Density/ W'm \\
\hline 1 & 2 & 10.860 \\
\hline 2 & 4 & 2.715 \\
\hline 3 & 6 & 1.207 \\
\hline 4 & 10 & 0.679 \\
\hline 5 & 14 & 0.636 \\
\hline 6 & 18 & 0.222 \\
\hline 7 & 22 & 0.090 \\
\hline 8 & 23.3 & 0.080 \\
\hline 9 & 26 & 0.064 \\
\hline
\end{tabular}

TABLE IV. Electromagnetic Radiation Measured Results

\begin{tabular}{|c|c|c|c|c|}
\hline \multirow[b]{2}{*}{$\begin{array}{c}\text { serial } \\
\text { number }\end{array}$} & \multirow{2}{*}{$\begin{array}{c}\text { Axial } \\
\text { Distance } \\
\text { from } \\
\text { Antenna/m }\end{array}$} & \multicolumn{2}{|c|}{ Measured Results } & \multirow{2}{*}{$\begin{array}{c}\text { Sum of } \\
\text { Power } \\
\text { Density } \\
/ \mathrm{W} \cdot \mathbf{m}^{-2}\end{array}$} \\
\hline & & $\begin{array}{c}\text { GSM Power } \\
\text { Density } / W \cdot m^{-2}\end{array}$ & $\begin{array}{c}3 G \text { Power } \\
\text { Density } / W \cdot m^{-2}\end{array}$ & \\
\hline 1 & 2 & 4.209 & 0.285 & 4.493 \\
\hline 2 & 4 & 1.240 & 0.295 & 1.535 \\
\hline 3 & 6 & 0.961 & 0.250 & 1.211 \\
\hline 4 & 10 & 0.250 & 0.083 & 0.333 \\
\hline 5 & 14 & 0.163 & 0.053 & 0.216 \\
\hline 6 & 18 & 0.096 & 0.034 & 0.130 \\
\hline 7 & 22 & 0.054 & 0.023 & 0.076 \\
\hline 8 & 26 & 0.034 & 0.013 & 0.048 \\
\hline
\end{tabular}

From the two tables, it could be drawn that, in single base station (WCDMA), there was large error between the predicted value and the monitoring value, less than $10 \mathrm{~m}$. In multi-systems base-station, there was large error between the predicted value and the monitoring value, less than $6 \mathrm{~m}$. Because of the electromagnetic radiation prediction model was based on the far field condition.

Simultaneously, according to Electromagnetic Radiation Standard: HJ/T 10.3-1996 《Guidelines on Management of Radioactive Environmental Protection Environmental Impact Assessment Methods and standards on Electromagnetic Radiation》 [9], the impact of a single project must be restricted in a number of GB 8702-88 limit, usually to $1 / 5$ of the power density as the environmental management target value, that was $8 \mu \mathrm{W} / \mathrm{cm} 2$.

From the table V, it could be seen that in single base station condition, the safe distance is about $15 \mathrm{~m}$. And in 
multi-systems base-station condition, the safe distance increased to about $22 \mathrm{~m}$.

TABLE V. Administrative Target Valve of Pubic Exposure

\begin{tabular}{|c|c|c|}
\hline $\begin{array}{c}\text { Frequency } \\
\text { Range/MHz }\end{array}$ & $\begin{array}{c}\text { Electric Field } \\
\text { Strength/ V } \cdot \mathbf{m}^{-\mathbf{1}}\end{array}$ & $\begin{array}{c}\text { Power } \\
\text { Density/W} \cdot \mathbf{m}^{-2}\end{array}$ \\
\hline $30 \sim 3000$ & $=5.4$ & $4 . / 5=8$ \\
\hline
\end{tabular}

\section{CONCLUSIONS}

Due to the working mechanisms and power controls of GSM, CDMA, WCDMA, TD-SCDMA system are not the same, in order to get better predicted results, it is necessary to pay attention to analyze the near-field conditions and power control of the various systems, and get a more accurate average transmit power value, in prediction of the shared base station electromagnetic radiation, in the future. Study on the sharing of electromagnetic radiation of mobile base station communication theory and computational methods, establishment of multi-systems base-station electromagnetic radiation prediction and evaluation model, and through the actual monitoring data to test the accuracy and practicability of the model. Multi-systems base-station electromagnetic radiation model has important direct sense for mobile communication base station radiation environmental management, design and construction, environmental impact assessment and prediction. And also it has real significance and far-reaching influence on the sustainable development, environment plan and protection of electromagnetic radiation of a city.

\section{ACKNOWLEDGMENT}

This work is supported by Chongqing Environmental Protection Agency Project (HT20120302-HB02) and the Chongqing Communication College Project (Research on dynamic sharing mechanism and evaluation optimization for spectrum resource in Multi-Wireless Sensor Network).

\section{REFERENCES}

[1] Chen Jiliang, Wen Ruchun. Space-time distribution \& attenuation feature about electromagnetic radiation of mobile communication base station. Shanghai Environmental Sciences, 2012, vol. 2, pp. 118 121.

[2] Zhang Tao. Mobile communication base station electromagnetic radiation protection from the correction algorithm research : http://d.g.wanfangdata.com.cn/Conference 7230884.asp.

[3] Shen Leyuan. Environmental Impact Assessment of GSM Network and 3G Network in Common Base Station of Mobile Communication. Pollution Control Technology, 2011, vol. 6, pp. 41-43.

[4] Fan Lei1, Pan Wei. Discussion on Safety Areas and Safety Distances of Electromagnetic Radiation at Multi- system Base Station. Radiation Protection. 2011, vol. 2, pp. 95-100.

[5] Wang Yuehua Yuan Yuming Zhou Baige. Analysis on the effects of electromagnetic radiation from WCDMA base station on ambient environment. Environment Engineering. 2012, vol. 1, pp.132-135.

[6] Wen Ruibiao. Impact of electromagnetic radiation on the surrounding environment of GSM mobile communication base station. Ecology and Environmental Sciences.2011, vol. 6-7, pp. 1158-1160.

[7] Gao Xiang, Liu Qunfang, Yao Yong. Analysis of theoretical prediction and field measurement about gsm mobile base station electromagnetic radiation protection level. Radiation Protection, 2011 vol. 1, pp. 45-49.
[8] Dan Rumeng. The Application of Mitigation Techniques for Limiting Human Exposure to EMFs in the Vicinity of Radio Communication Stations. SAFETY \& EMC 2010, vol.1, pp. 15-19.

[9] ITU-T Recommendation K.70 : 2007 Mitigation techniques to limit human exposure to EMs in the vicinity of radiocommunication stations. 2007 